\title{
Temporal evolution of photorefractive double phase-conjugate mirrors
}

\author{
Mordechai Segev, Doruk Engin, and Amnon Yariv \\ California Institute of Technology, 128-95, Pasadena, California 91125 \\ George C. Valley \\ Hughes Research Laboratories, 3011 Malibu Canyon Road, Malibu, California 90265 \\ Received April 6, 1993

\begin{abstract}
We present wave-optics calculations of the temporal and spatial evolution from random noise of a double phaseconjugate mirror in photorefractive media that show its image exchange and phase-reversal properties. The calculations show that for values of coupling coefficient times length greater than two the process exhibits excellent conjugation fidelity, behaves as an oscillator, and continues to operate even when the noise required for starting it is set to zero. For values less than two, the double phase-conjugation process exhibits poor fidelity and disappears when the noise is set to zero.
\end{abstract}

The discovery of double phase conjugation by Weiss et al. ${ }^{1}$ has stirred much controversy concerning the temporal and spatial processes by which two mutually incoherent input beams become phase conjugates of each other. Originally, many researchers thought the process apocryphal since two incoherent laser beams cannot usually write a stationary interference pattern with each other. Weiss et al. realized, however, that both beams fan, and, since they share the same holographic medium, an effective interaction between them is produced through the scattering off each other's gratings. In particular, the highest gain for this process occurs when the two beams become counterpropagating phase conjugates of each other (i.e., the beams exchange slowly varying amplitude and phase profiles with a sign change on the phase). It has been shown ${ }^{1}$ that when the input beams to the double phase-conjugate mirror (DPCM) carry pictorial information, they exchange this information with essentially no cross talk or print through.

Previous analytical research on the DPCM is contradictory. A one-dimensional analysis, which by its nature cannot address issues of conjugation fidelity or replication quality, suggests that the DPCM is an oscillator with a threshold of coupling coefficient times length equal to two. ${ }^{1}$ Simplified two-dimensional analyses ${ }^{2}$ neglect the effects of diffraction, phase matching, and the nonlocal nature (i.e., the refractive-index perturbation at a point is generated solely by the field amplitudes at exactly the same point) of the photorefractive effect and are therefore inapplicable (as shown in Ref. 3, these simplifications cannot be made for image-bearing beams or when the phase-conjugation process starts from noise).

Here we present two-dimensional wave-optics calculations of the temporal and spatial evolution of the DPCM for beams fully overlapping inside the crystal (see the inset in Fig. 4 below). We find that the process has two properties that are characteristic of an oscillator. First, there is a well-defined threshold in conjugation fidelity at coupling-coefficient times length equal to two. Second, for a coupling coefficient times length greater than two, one can turn off the noise that seeds the DPCM, and the DPCM remains substantially unchanged, whereas for values less than two it disappears.

We formulate the problem of two-beam propagation in a photorefractive medium in the terms of the plane-wave expansion technique used recently used to describe stimulated scattering, ${ }^{4}$ fanning, ${ }^{5,6}$ and photorefractive solitons. ${ }^{7}$ We neglect interactions between orthogonal components (anisotropic scattering), assume two monochromatic nearly counterpropagating incident beams of arbitrary input amplitude profiles, and include only two spatial dimensions. The total electric field $E(x, z, t)$ of the two optical beams of the same average frequency $\omega$, counterpropagating in the $z$ direction, is

$$
\begin{aligned}
E(x, z, t)= & \frac{1}{2}\left[A_{1}(x, z, t) \exp (i k z-i \omega t)\right. \\
& \left.+A_{2}(x, z, t) \exp (i k z+i \omega t)+\text { c.c. }\right] .
\end{aligned}
$$

We expand each beam in plane waves:

$$
\begin{array}{r}
A_{1}(x, z, t)=\sum_{m} a_{m}(z, t) \exp [-i k \epsilon m(x+\epsilon m z / 2)], \\
A_{2}(x, z, t)=\sum_{m} b_{m}(z, t) \exp [i k \epsilon m(x+\epsilon m z / 2)],
\end{array}
$$

where $A_{1}(x, z, t)$ and $A_{2}(x, z, t)$ are the slowly varying parts of the field, $x$ is the transverse coordinate, $k=\omega n_{b} / c$ is the optical wave number, $n_{b}$ is the background refractive index in the crystal, $\epsilon$ is the angular separation of the plane waves, and $a_{m}(z, t)$ and $b_{m}(z, t)$ are the expansion coefficients. Note that in practice the interacting beams are nearly counterpropagating ( $173^{\circ}$ in Ref. 1 ) and that we account for the angular difference in the envelopes 
$A_{i}$. The two beams are assumed to be incoherent with each other (i.e., we assume that $\left\langle A_{1} A_{2}{ }^{*}\right\rangle=0$ ), yet the index perturbations formed by each beam contribute to the scattering of both beams. Since there is no direct interaction between the beams, the amplitudes $A_{i}$ separately obey the nonlinear paraxial wave equations (assuming negligible absorption)

$$
\begin{aligned}
& \frac{\partial A_{1}}{\partial z}-\frac{i}{2 k} \frac{\partial^{2} A_{1}}{\partial x^{2}}=\frac{i k}{n_{b}} \delta n(x, z, t) A_{1}, \\
& \frac{\partial A_{2}}{\partial z}+\frac{i}{2 k} \frac{\partial^{2} A_{2}}{\partial x^{2}}=\frac{-i k}{n_{b}} \delta n(x, z, t) A_{2},
\end{aligned}
$$

where the same refractive-index perturbation $\delta n(x, z, t)$ that appears in both equations is produced by both beams separately and evolves in time as

$$
\begin{aligned}
& \frac{\partial \delta n}{\partial t}+\left(1+\left|A_{1}\right|^{2}+\left|A_{2}\right|^{2}\right) \delta n=i \sum_{m, n} \gamma_{m n}\left\{a_{m} a_{n}{ }^{*}\right. \\
& \times \exp \left[-i k(m-n) x \epsilon-i k \epsilon^{2}\left(m^{2}-n^{2}\right) z / 2\right]+b_{m} b_{n}{ }^{*} \\
& \left.\quad \times \exp \left[i k(m-n) x \epsilon+i k \epsilon^{2}\left(m^{2}-n^{2}\right) z / 2\right]\right\}
\end{aligned}
$$

[for the rigorous derivation of Eq. (6) see App. A of Ref. 4], where $\gamma_{m n}$ are the coupling coefficients between the individual plane waves ${ }^{5}$ (note that energy conservation, when no absorption gratings are present, is manifested in $\left.\gamma_{m n}=-\gamma_{n m}{ }^{*}\right)$. Here we assume that $\gamma_{m n}$ are real, since the DPCM is generated through an energy-transfer process caused by the nonlocal (or real) part of the coupling coefficients. In Eqs. (1)-(6) the field amplitudes, $A_{i}, a_{n}$, and $b_{n}$, are given in units of the square root of the equivalent dark irradiance and time is in units of the dielectric relaxation time evaluated at the equivalent dark irradiance.,6 To make the calculations tractable ${ }^{6}$ we define $I_{0}$ as the spatial average of $\left(1+\left|A_{1}\right|^{2}+\left|A_{2}\right|^{2}\right)$ and substitute this into Eq. (6). The refractive-index perturbation $\delta n(x, z, t)$ my also be expanded in the form

$$
\begin{aligned}
\delta n(x, z, t)= & \sum_{m, n} n_{m, n}(z, t) \exp [-i k(m-n) x \epsilon \\
& \left.-i k \epsilon^{2}\left(m^{2}-n^{2}\right) z / 2\right] .
\end{aligned}
$$

Substitution of Eqs. (2) and (7) into Eq. (4), multiplication by the factor $\exp (i k p x \epsilon)$, and integration over $x$ yield

$$
\frac{\partial a_{p}}{\partial z}=\frac{i k}{n_{b}} \sum_{m, n} n_{m n} a_{p+n-m} \exp \left(-i k \epsilon^{2} \Delta_{1} z / 2\right) .
$$

Similar operations on Eq. (5) lead to

$$
\frac{\partial b_{p}}{\partial z}=\frac{-i k}{n_{b}} \sum_{m, n} n_{m n} b_{p-n+m} \exp \left(i k \epsilon^{2} \Delta_{2} z / 2\right) .
$$

Similarly, Eq. (6) is transformed into

$$
\frac{\partial n_{m n}}{\partial t}+I_{0} n_{m n}=\gamma_{m n}\left(a_{m} a_{n}^{*}+b_{m}{ }^{*} b_{n}\right),
$$

where $\Delta_{1}=2(m-n)(m-p), \Delta_{2}=2(n-p)(n-m)$, and $k \epsilon^{2} \Delta / 2$ is the momentum mismatch between a specific plane wave $a_{p}$ (or $b_{p}$ ) and the grating $n_{m n}$.
Inclusion of only the phase-matched terms in Eqs. (8) and (9) yields (recall that $\gamma_{m m}=0$ ):

$$
\begin{aligned}
& \frac{\partial a_{p}}{\partial z}=\frac{i k}{n_{b}} \sum_{n} n_{p n} a_{n}, \\
& \frac{\partial b_{p}}{\partial z}=\frac{-i k}{n_{b}} \sum_{n} n_{n p} b_{n} .
\end{aligned}
$$

Note the solution ${ }^{8}$ that yields maximal scattering from mutual gratings: $a_{p}=b_{p}{ }^{*}$ for all $p$, which is not necessarily reached for arbitrary images, initial conditions, and coupling coefficients.

In our calculations, we use parameters typical of $\mathrm{BaTiO}_{3}$ that provided a good match between steadystate fanning observations and calculations. ${ }^{5,6}$ Angles are measured with respect to the $+c$ axis, and 31 plane waves are used to describe the transverse dependence (angular separation of half a degree). We have solved the equations numerically by using the split-step methods, ${ }^{4}$ and we have checked the calculations by doubling the number of steps in $z$ and $t$ and in the transverse resolution in $x$ (namely, reducing the angular separation to $0.25^{\circ}$ ). As found in Ref. 6, our calculations show that the non-phasematched terms are important only for propagation distances of a few optical wavelengths, and hence all our results are based on numerical solution of Eqs. (10) and (11).

We calculate the full temporal evolution of the DPCM and show the steady-state results in Figs. 1 and 2 and the phase-conjugation fidelity versus time for several different coupling levels in Fig. 3. The total intensity is $I_{0}=1$, and the intensity ratio of the input beams is 0.87 . The average coupling coefficient for the transmission gratings geometry of

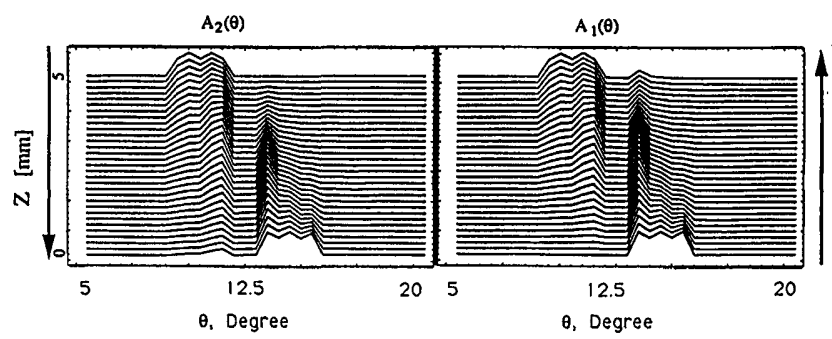

Fig. 1. Steady-state spatial evolution of the plane-wave amplitudes for a DPCM in a photorefractive crystal.
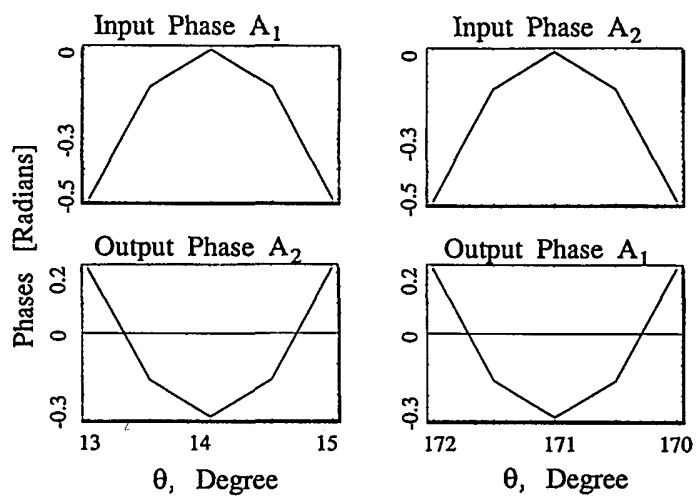

Fig. 2. Steady-state input and output phases for the interacting beams at angles corresponding to the location of pictorial information. 


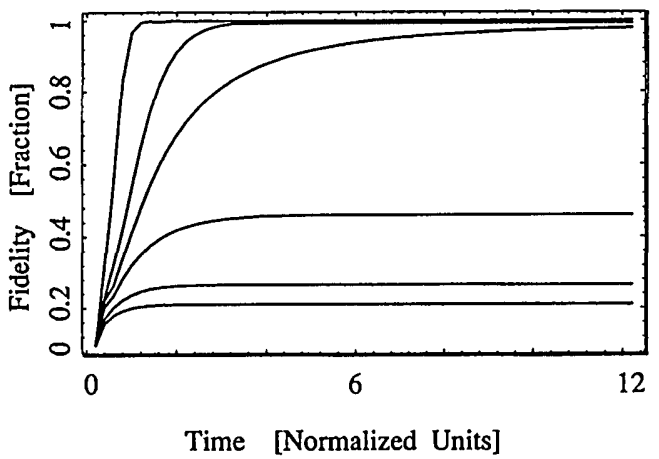

Fig. 3. Phase-conjugation fidelity as a function of time for several gain levels (from the top to the bottom curves): $\gamma_{0} L \approx 4.9,2.46,2.05,1.64,1.23,0.98$.

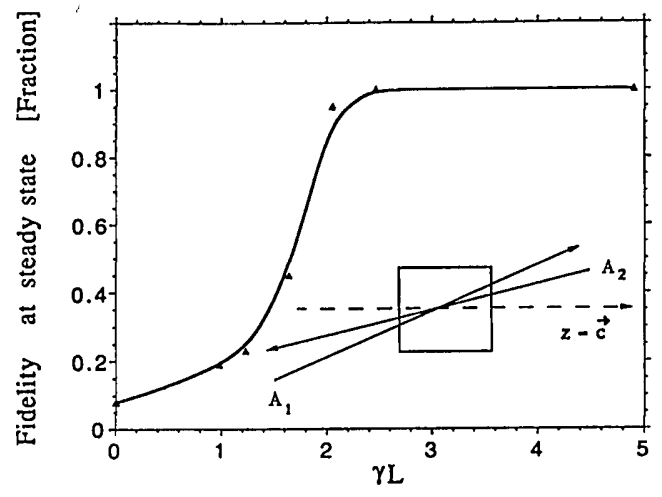

Fig. 4. Phase-conjugation fidelity at steady state as a function of coupling coefficient times length $\gamma_{0} L$.

Fig. 1 is approximately $\gamma_{0} \approx 9.83 \mathrm{~cm}^{-1}$. As in the experimental observation, ${ }^{1}$ the process builds up from noise scattering (fanning), and therefore we introduce an initial noise level of $10^{-4} I_{0}$ (i.e., $a_{n}, b_{n}=10^{-2}$ for every $n$ at $z=0$ and $z=5 \mathrm{~mm}$ ), with a random phase. In Fig. 1 we plot the steady-state spatial evolution of the plane-wave amplitudes in the Fourier (spatialfrequency) plane for several planes perpendicular to the propagation distance $z$. The input beams are at the top left $\left(A_{2}\right)$ and bottom right $\left(A_{1}\right)$, and the arrows indicate the direction of propagation for the two beams (the input profiles were completely arbitrary). The left-hand plot shows the propagation of $A_{2}$ and its transformation (like some drawings of Escher ${ }^{9}$ ) into the phase conjugate of $A_{1}$, and the right-hand plot shows the similar evolution for $A_{1}$.

Figure 2 shows the steady-state input and output phases for both beams, in the vicinity of their pictorial information, where the inversion of the phase manifests the phase-conjugation properties of the output beams. Note that the phase-reversed image acquires an additional bias (transversely uniform) phase of $-0.3 \mathrm{rad}$, which is maintained for all gain levels. For lower gains, a deterioration in the phase-conjugation fidelity appears, manifested as differences in the small features of the Fourier-plane amplitudes, which correspond to large-scale distortions in the real plane. Figure 3 shows the phase conjugation fidelity $\mathrm{CF}$, defined by

$$
\mathrm{CF}=\sum_{m} a_{m} b_{m} /\left(\sum_{m}\left|a_{m}\right|^{2} \sum_{m}\left|b_{m}\right|^{2}\right)^{1 / 2}
$$

as a function of time for several gain levels. Figure 4 shows the phase-conjugation fidelity at steady state as a function of gain. There is a fairly sharp threshold at $\gamma_{0} L \approx 2$ for the phase-conjugation process, and good fidelity can be obtained in steady state at or above this threshold. To verify that the DPCM is an oscillator, we set the noise source term to zero after reaching steady state. For coupling coefficients times length less than two, we observed that the conjugate wave decreases to near zero. For values greater than two, the conjugate wave remains essentially unchanged. We have also evaluated this effect for large input beam ratios and found that the threshold increases dramatically, as predicted. ${ }^{1}$ We have also changed the scaling factor for the noise for values of $\gamma_{0} L$ slightly above threshold and found that the fidelity is almost insensitive to the noise level. Slightly below threshold, however, the fidelity is very sensitive to the level of noise.

In conclusion, we present a model for the temporal and spatial evolution from random noise of the DPCM in photorefractive materials and demonstrate image exchange and phase reversal between the beams. We conclude that the DPCM is indeed an oscillator, since high phase-conjugation fidelity can be obtained only above a well-defined gain threshold.

We acknowledge the enlightening comments of the reviewers of this Letter.

Note added in proof: In calculations performed after this Letter was accepted, we find that the threshold value is not necessarily equal to 2 , in general, but depends on the spatial profile of the input beams and decreases with decreasing feature size in the information borne on the beams.

\section{References}

1. S. Weiss, S. Sternklar, and B. Fischer, Opt. Lett. 12, 114 (1987); Appl. Phys. Lett. 50, 483 (1987); Opt. Eng. 26, 423 (1987).

2. A. A. Zozulya, Opt. Lett. 16, 545 (1991); V. V. Eliseev, V. T. Tikhonchuk, and A. A. Zozulya, J. Opt. Soc. Am. B 8, 2497 (1991); N. V. Bobodaev, V. V. Eliseev, L. I. Ivleva, A. S. Korshunov, S. S. Orlov, N. M. Polozkov, and A. A. Zozulya, J. Opt. Soc. Am. B 9, 1493 (1992); K. D. Shaw, Opt. Commun. 90, 133 (1992); 94, 458 (1992).

3. B. Ya. Zel'dovich, N. F. Pilipetsky, and V. V. Shkunov, Principles of Phase Conjugation (Springer-Verlag, Berlin, 1985), Chaps. 2-5.

4. G. C. Valley, J. Opt. Soc. Am. B 9, 1440 (1992).

5. M. Segev, Y. Ophir, and B. Fischer, Opt. Commun. 77, 265 (1990).

6. M. Segev, D. Engin, A. Yariv, and G. C. Valley, Opt. Lett. 18, 956 (1993).

7. M. Segev, B. Crosignani, A. Yariv, and B. Fischer, Phys. Rev. Lett. 68, 923 (1992); B. Crosignani, M. Segev, D. Engin, P. DiPorto, A. Yariv, and G. Salamo, J. Opt. Soc. Am. B 10, 446 (1993).

8. M. Segev and A. Yariv, Opt. Lett. 16, 1938 (1991).

9. M. C. Escher and J. L. Locher, The World of M. C. Escher (Abrams, New York, 1971), p. 84. 